\title{
MICROCONTROLLER BASED SMART WEAR FOR DRIVER SAFETY
}

\author{
Abhinav Anand ${ }^{1}$, Kumar Harsh ${ }^{2}, K_{\text {Kushal Kumar }}{ }^{3}$, Sourav Gouthi $^{4}$ \\ ${ }^{1}$ Department of Instrumentation Technology, Dayananda Sagar College of Engineering, Bangalore, India \\ ${ }^{2}$ Department of Instrumentation Technology, Dayananda Sagar College of Engineering, Bangalore, India \\ ${ }^{3}$ Department of Instrumentation Technology, Dayananda Sagar College of Engineering, Bangalore, India \\ ${ }^{4}$ Asst. Professor Ekta Maini, Department of Instrumentation Technology, Dayananda Sagar College of Engineering, \\ Bangalore, India
}

\begin{abstract}
An accident is a specific, unexpected, unusual and unintended external action which occurs in a particular time and place, with no apparent and deliberate cause but with marked effects. Carelessness of the driver is the major factor of such accidents. The traffic authorities give a lot of instructions to the vehicle operators. But many of them do not obey the rules. Nowadays most of the countries are forcing the motor riders to wear the helmet and not to use the vehicles when the person is in drunken condition. But still the rules are being violated by the users. India has the largest number of road accidents in the world out of which $22.4 \%$ of victims are occupants of two wheelers, $30 \%$ of road accidents victims die at crash site, $14 \%$ in ambulance and rest in hospital. Our proposal is to reduce the number of deaths which occur at crash site and on the way to the hospital due to delay in Emergency Trauma Care Services.
\end{abstract}

Keywords- accident, carelessness, helmet, drunken driving, two wheelers

\section{INTRODUCTION}

Providing safety to a person while riding the bike is of prime concern. One of the way to do this is by making it mandatory to wear helmet while riding a bike. This is difficult to implement as every time the concerned people can't keep an eye on everybody. So detecting whether the driver has worn a helmet or not, as well whether he has consumed alcohol or not is the main problem. The system what we have planned to design provides solution to this problem. The system makes it mandatory for the rider to wear helmet before starting the vehicle and also he shouldn't have consumed alcohol. If the rider fail to do so then the vehicle cannot be started. This system also provides security to the vehicle as every bike will have a unique helmet and without which a person fails to start that particular bike. This project includes a helmet body and an integrated electronic system disposed in the helmet body. It is operated through a wireless control system. The components of the electronic system are sufficiently small and rugged for use in the helmet, ensuring that the helmet is lightweight and durable. Moreover, the components are spaced about the helmet to provide even weight distribution to promote overall balance and safety

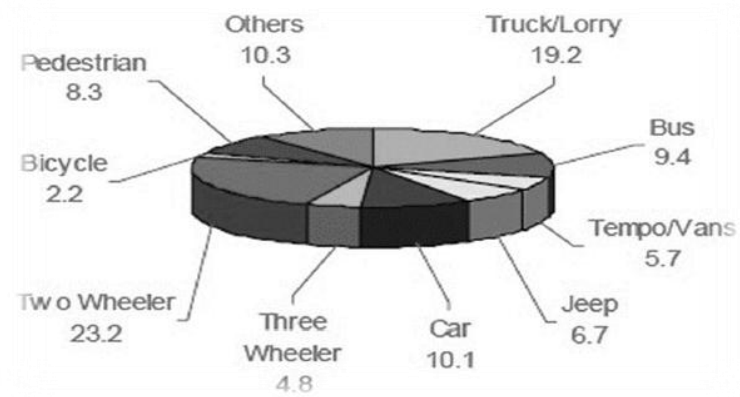

Fig.1 Accidents occurrence on different vehicles

\section{PROPOSED SYSTEM}

\subsection{Helmet Detection:}

The circuit in each helmet is designed in such a manner that the bike won't start until the rider wears the helmet. This can be done with the help of an earlobe sensor which will detect the IR radiations transmitted from the human body to ensure that the helmet is worn by the rider.

\subsection{Accident Detection:}

In our busy day to day life, due to lack of quality of the parts of our vehicles or due to our hurry or due to some other reason, accident may take place. Even though we have advanced medical equipment, we are unable to save many of lives. Why because we are not getting information about the accident location in right time. Our system works for this.

\subsection{Alcohol Detection:}

This relatively low-cost and easy to build project detects the presence of alcohol of the people who are drunk and drive the vehicle. On sensing the alcohol, a signal will be sent to the controller and an alarm is activated.

\subsection{Speed Control:}

In this application we will be monitoring the areas in which the vehicle will be passing. On entering any cautionary areas like schools, the speed of the bike will be controlled to a predefined limit 


\section{BLOCK DIAGRAM}

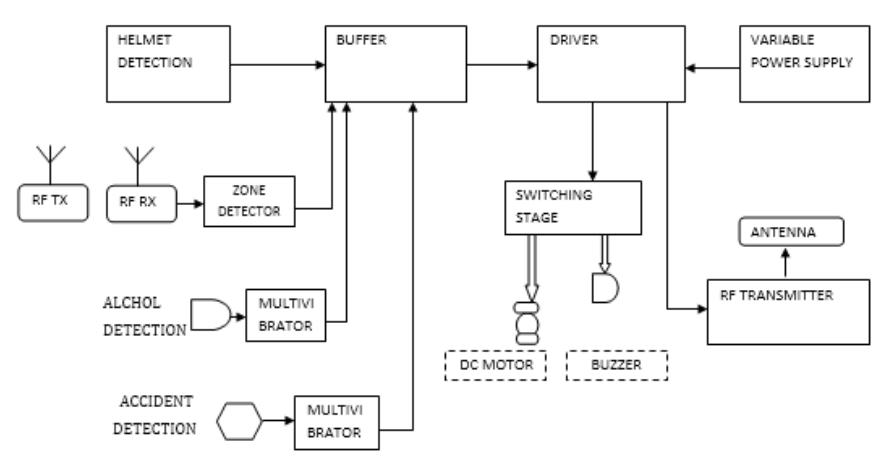

Fig.2. Helmet section of the safety gear

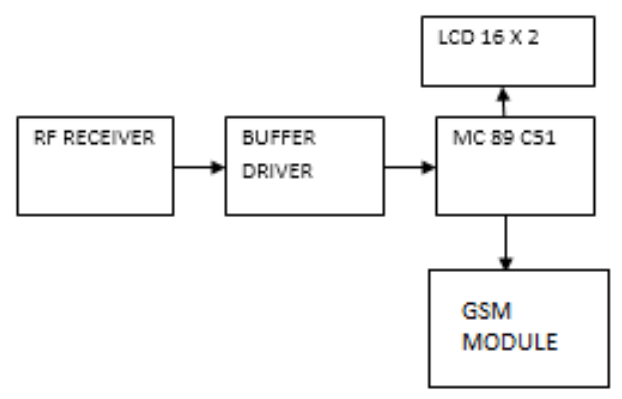

Fig.3. Section of the safety gear in the bike

Earlobe Sensor: The earlobe sensor is used to detect weather the rider is wearing the helmet. It is an Infrared sensor which detects radiation that is being emitted by the human body.

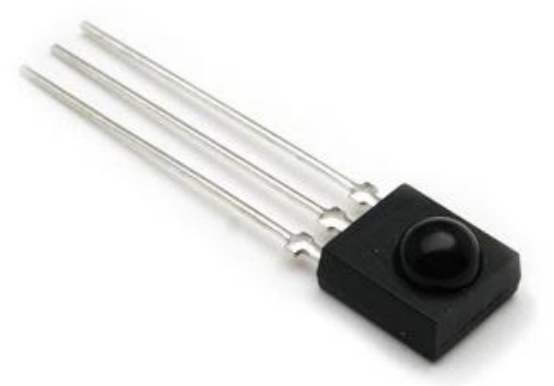

Fig.4. An IR Sensor

Alcohol Sensor: MQ-3 gas detector (alcohol sensor) is suitable for detecting alcohol content from the breath. So it can be placed just below the face defend and above the additional face protection. The surface of the sensor is sensitive to various alcoholic concentrations. It detects the alcohol from the rider's breath; the resistance value drops leads to change in voltage (Temperature variation occurs).Generally the illegal consumption of alcohol during driving is $0.08 \mathrm{mg} / \mathrm{L}$ as per the government act. Except for demonstration purpose, we have a tendency to program the drink limit as $0.04 \mathrm{mg} / \mathrm{L}$. Threshold will be adjusted mistreatment exploitation.

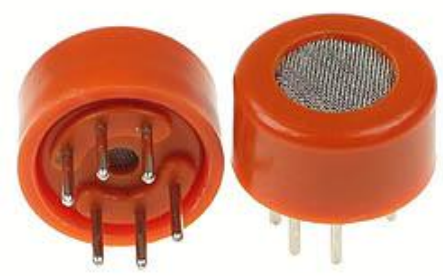

Fig.5.Alcohol sensor

Accident Detection: Accident occurrence will be detected by the piezoelectric sensor and further this information is given to microcontroller. The GPS will be tracking the location of the vehicle at where the accident has occurred. GPS can get the graphical location of the vehicle. The location values are given to microcontroller. Controller gives this information to GSM module. By using GSM we can send the message to family members, emergency medical service and nearest hospital.

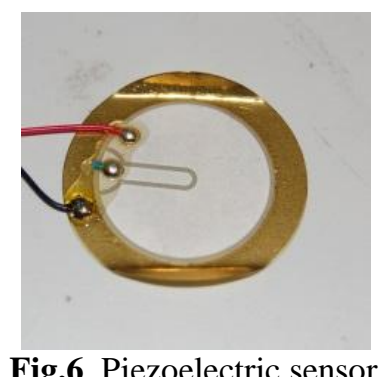

RF Transmitter: This is 2-channel Radio Frequency Transmitter specially tuned with its RF Receiver part in carrier frequency. Each zone are set with one channel and transmits their presence to moving vehicle's RF Receiver unit.

RF Receiver: This is also a 2-channel RF Receiver specially tuned with its counterpart RF Transmitter in carrier frequency. When vehicle enters into any zone that zones RF signals are received by this unit. Thus depend upon the channel signals it receives from transmitting end that channel output of RF Receiver goes HIGH. This HIGH signal is fed to Microcontroller chip through Buffer \& Driver and Switching stage for further processing.

Buffer \& Driver: The Buffer provides unit gain amplification to inputted signal and fed it to Driver for driving relay. The Driver is power amplifier, which provides high current and voltage to relay coil for proper switching action.

Switching Stage: This stage is comprised of suitable voltage rated relays energized by $\mathrm{HIGH}$ signal given to respective Buffer \& Driver stage.

Microcontroller Chip: The chip has software module inherited in its memory and which scans continuously its input port pins for HIGH signal. When RF Receiver sends any HIGH signal that is fed to Buffer \& Driver and energizes one relay. This relay's N/O [Normally Open] pins 
come into action and in turn supplies exact +5 Volts to input port pin of chip. This is necessary because chip accepts TTL compatible signals [i.e., $\mathrm{LOW}=0 \mathrm{~V}$ and $\mathrm{HIGH}=$ exact +5 $\mathrm{V}]$ at its input port. After receiving any HIGH signal at its input port, software module identifies the zone by HIGH input pin number and accordingly generates alert message and control signal to output port. The alert message is sent to output port where LCD Module is connected for display purpose. The control signal is fed to Buffer\& Driver and Switching Stage for execution of control signal.

LCD Module: This is a output device connected to output port of chip and displays alert message depends upon the zone in which vehicle enters.

Variable Power Supply: This specially designed power supply provides two voltages, Normal and Low to control the loads.

Power Supply Unit: The system needs regulated power supply which provides necessary voltages for proper working of its blocks. This unit is specially designed to gives out all necessary voltage levels required for working of all blocks

\section{FLOWCHART REPRESENTATION}

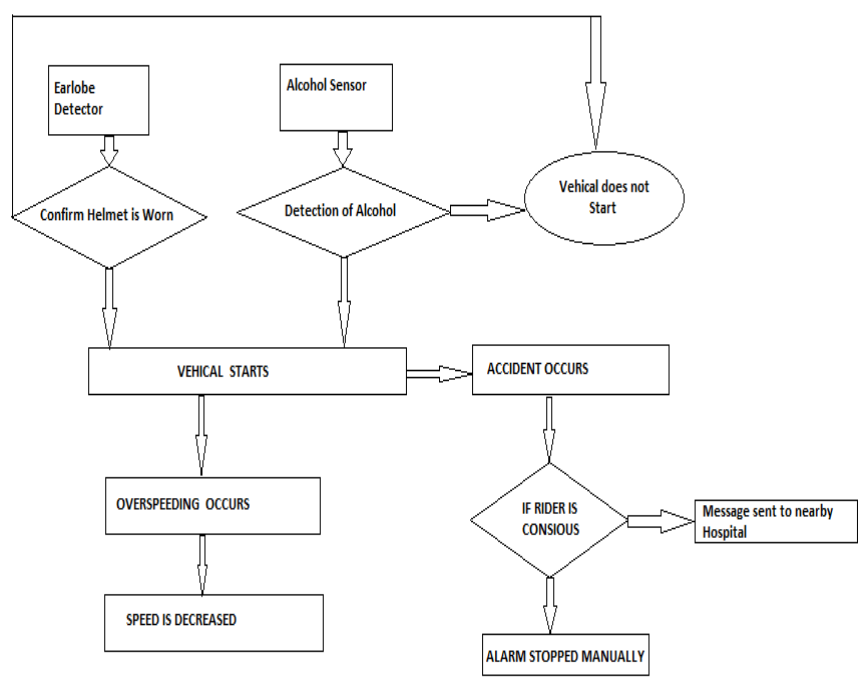

Fig.7. Flowchart representation of the proposed system

\section{RESULT}

Some of the snapshots of the result are shown below

\section{WEAR HELMET IGHITIOH DFF}

Fig.8. LCD module displaying the message to wear helmet

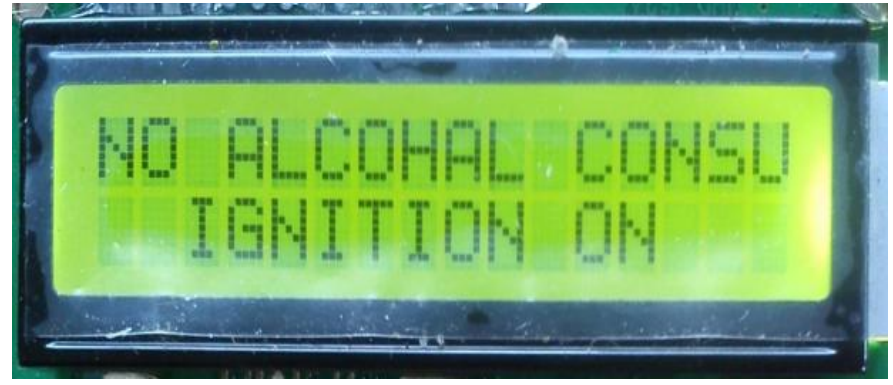

Fig.9.LCD showing no alcohol is consumed by driver

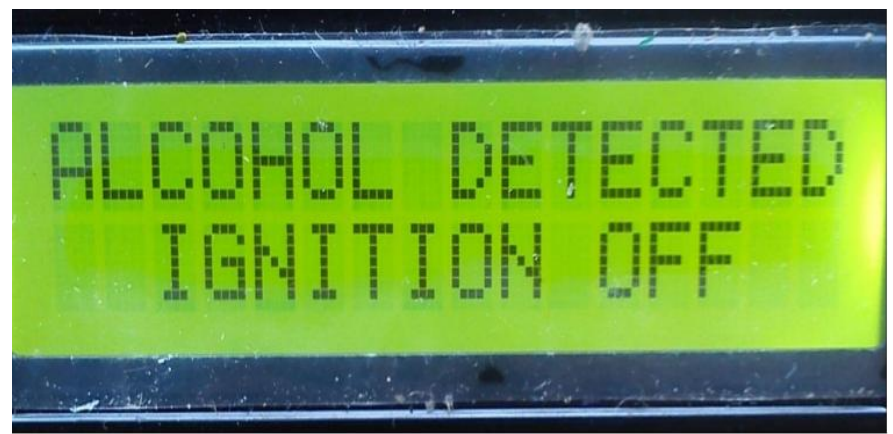

Fig.10.LCD showing alcohol is consumed by the driver

Fig.9. and Fig.10. shows that when the alcohol consumed by the driver is above a pre-set limit, the ignition will not turn on.

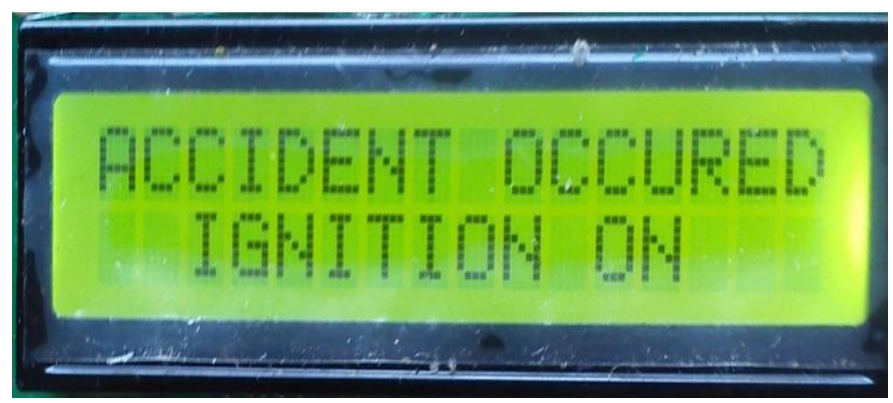

Fig.11.Accident occurred message displayed on LCD

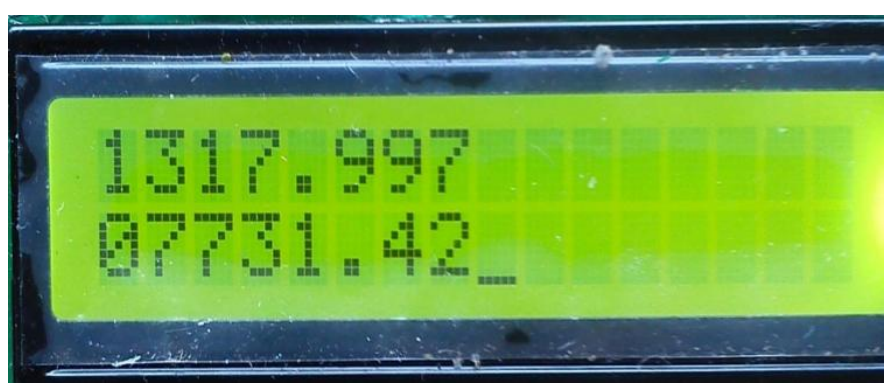

Fig.12.Latitude and longitude values of the place where accident occurred

Fig.11. and Fig.12 shows the message which will be displayed in case of an accident. In case of an accident a alarm system will be generated which can be manually turned off. If it is not turned off within a specified time, the location where the accident has occurred will be sent to the nearest hospital so that the injured driver can get the necessary medical attention as soon as possible 


\section{CONCLUSION}

This project can be used to secure and avoid road accidents. It is easy to install an easy to operate. Moreover, manpower can be saved by the use of auto detecting circuits. The main aim of this project is to encourage the people to wear the helmet and to avoid the road accidents.This project ensures safety of riders and prevention of road accidents to some extent. The helmet incorporates a very simple and cost effective technology. Also as a future scope it can be combined with many existing technologies to provide guidance and on road track facility

\section{REFERENCES}

[1]. M. Pieve, F. Tesauri, and A. Spadoni, "Mitigation accident risk in powered two wheelers domain: Improving effectiveness of human machine interface collision avoidance system in two wheelers," in Proc. 2nd. Conf. Human Syst. Interact., Catania, Italy, May 21-23, 2009, pp. 603-607

[2]. E.D.Bekiaris, A.Spadoni, and S.I.Nikolaou, "SAFERIDER project: New safety and comfort in powered two wheelers," in Proc. 2nd Conf. Human Syst. Interact., Catania, Italy, May 21-23, 2009, pp. 600-602.

[3]. M.Cellario,"Human-centered intelligent vehicles: Toward multimodal interface integration," IEEE Intell. Syst., vol. 16, no. 4, p. 78, Jul. 2001. 